\title{
Les Albums plurilingues ÉLODiL pour soutenir l'enseignement à distance
}

\author{
Catherine Gosselin-Lavoie \\ Université de Montréal \\ Canada \\ Catherine Maynard \\ Université Laval \\ Canada \\ Françoise Armand \\ Université de Montréal \\ Canada
}

\begin{abstract}
Lorsque la langue de scolarisation est différente de celle·s parlé e.s à la maison, son apprentissage représente un défi supplémentaire. C'est le cas pour certains élèves bi/plurilingues en émergence, scolarisés dans les écoles québécoises francophones. À titre d'exemple, en 2019, à Montréal, ville où se trouve la plus forte concentration de ces élèves, ils représentaient $43,1 \%$ des effectifs du réseau public, du préscolaire au secondaire (Comité de gestion de la taxe scolaire de l'ile de Montréal (CGTSIM), 2020). Parmi près de 200 langues maternelles autres que le français ou l'anglais déclarées par les familles, l'arabe, l'espagnol, le créole, le chinois et le tagalog/filipino sont les plus représentées. Ce défi revêt d'autant plus d'importance dans le contexte de la pandémie de Covid-19, qui a forcé de nombreuses écoles à fermer leurs portes, réduisant ainsi pour plusieurs élèves les contacts avec la langue, nécessaires au développement des compétences en langue seconde (Swain,1985). Afin de soutenir l'enseignement à distance, un effort collectif a été initié par le ministère de l'Éducation et de l'Enseignement Supérieur (MEES) du Québec (conception du site L'École Ouverte) et les centres de services scolaires (création de trousses pédagogiques) pour mettre à disposition des enseignant $\cdot e \cdot s$, des élèves et de leurs parents des outils pédagogiques et des ressources ludiques.
\end{abstract}

Dans le désir de contribuer à cet effort dans le domaine de l'apprentissage de la lecture et avec un souci particulier de favoriser l'ouverture à la diversité linguistique et la reconnaissance des répertoires linguistiques ${ }^{1}$ des élèves et de leurs familles, l'application les Albums plurilingues ÉLODiL ${ }^{2}$ (Armand, Gosselin-Lavoie et Maynard, 2018) a été rendue accessible gratuitement à l'intention des enseignant'e.s et des familles à travers le Canada ${ }^{3}$. Cette application regroupe 11 albums de littérature jeunesse en français, édités par deux maisons d'éditions québécoises (Les 400 Coups et La Courte échelle) et traduits, à l'écrit et à l'oral, dans plus d'une vingtaine de langues (listées plus loin). Elle a été développée dans le cadre d'une recherche-action qui avait pour objectif de soutenir, en classe ordinaire, le développement langagier à l'oral et à l'écrit d'enfants de maternelle (cinq ans) en milieu pluriethnique et plurilingue, au moyen de la littérature de jeunesse plurilingue et dans une perspective de collaboration école-famille (Armand et coll., 2017-2021).

Lors de la mise en place de cette recherche-action, les dix enseignant $\cdot e \cdot s$ qui exploitaient en classe l'application visaient, chez les enfants, par différentes activités spécialement conçues autour de 7 des 11 albums ${ }^{4}$, le développement de la compréhension orale de récits, des habiletés narratives, du vocabulaire et des concepts de l'écrit ainsi que l'ouverture à la diversité linguistique. Lors des exploitations des albums en classe, les enseignant $\cdot e \cdot s$ étaient invité $\cdot e \cdot s$ à nommer explicitement les langues au fur et à mesure qu'elles ou ils lisaient les albums, en français et/ou dans une ou plusieurs autres langues, et à inciter les enfants à s'exprimer sur ces langues (Dans quelle langue sommes-nous en train de lire l'histoire ? Avez-vous déjà entendu cette langue? Quand ? Où ? Connaissez-vous des personnes qui parlent cette langue?). Les enseignant·e·s ont aussi animé des activités sollicitant et développant chez les enfants des habiletés d'écoute, de discrimination auditive, d'observation et de comparaison entre les langues. Grâce à la variété de langues de l'application, transcrites par des systèmes d'écriture aux caractéristiques hétérogènes (alphabétiques ou non ; différents alphabets [par exemple, latin, grec, arabe] ; sens de l'écriture), les enseignant $e \cdot s$ pouvaient aussi poursuivre avec les enfants la familiarisation avec les concepts de l'écrit (notion de lettre et de mot, orientation de l'écrit et sens de la lecture, etc.) en s'appuyant sur des comparaisons entre les langues.

Durant la recherche-action, réalisée avant la pandémie, l'établissement d'une collaboration école-famille a permis aux parents des dix classes ciblées d'avoir accès à l'application, à distance, afin d'explorer de façon ludique les albums plurilingues et de poursuivre les apprentissages à la maison. Des ateliers, mis en place avec la collaboration de l'organisme communautaire J'apprends avec mon enfant (JAME) ${ }^{5}$, soutenaient les familles 
dans leur utilisation de cette application. Des recherches ont démontré que, lorsque les milieux éducatifs proposent des activités autour de la littératie qui permettent aux parents de participer activement, des effets bénéfiques sont perçus sur les apprentissages dans la langue maternelle et/ou dans la langue de scolarisation (Rodriguez-Valls, 2009). Une participation active peut se traduire entre autres par la mise en place d'approches faisant intervenir leurs connaissances et leurs compétences multiples, dont font partie leurs ressources linguistiques. II s'agit de mettre l'accent sur les approches plurilingues de la littératie, aussi nommées littératies multilingues (Hornberger, 2001), qui impliquent la légitimation du bagage culturel et linguistique des élèves (Armand et coll., 2016 ; Gosselin-Lavoie et Armand, 2015).

C'est dans cette optique que nous avons conçu l'application les Albums plurilingues ÉLODiL afin de réduire, en milieu pluriethnique et plurilingue, les frontières entre la langue de l'école et les langues des familles et, ainsi, de créer des situations de bilinguisme additif (Lambert, 1975) où les milieux scolaire et familial de l'enfant reconnaissent et tirent tous deux profits des ressources présentes dans son répertoire linguistique. Plus largement, cette application s'adresse aux enseignant $\cdot e \cdot s$ et aux parents qui souhaitent soutenir l'entrée dans l'écrit et favoriser, dans une perspective d'éducation interculturelle, l'ouverture à la diversité linguistique et culturelle de tous les enfants. Dès le mois d'avril 2020, l'annonce de l'accès gratuit à l'application sur la page Facebook professionnelle ÉLODiL et le large partage de l'information à travers différents réseaux (Bibliothèques publiques de la ville de Montréal, Faculté des sciences de l'éducation de l'Université de Montréal, centres de services scolaires, organisme JAME, etc.) a permis à un plus grand nombre d'enseignant·e.s et de parents de pouvoir en bénéficier.

Dans le cadre de cet article, nous exposons les fondements qui sous-tendent l'application et décrivons les modalités de sa conception. Nous présentons ensuite des données sur sa fréquentation et son utilisation à travers le Canada $^{6}$ ainsi que sur son appréciation par des enseignant·e·s (et quelques parents) qui l'ont utilisée durant les premiers mois de l'accès ouvert.

\section{Les Albums plurilingues ÉLODiL : fondements et description}

\section{Les albums de littérature jeunesse}

Figurant dans les programmes de formation de l'école québécoise (PFÉQ) (Éducation préscolaire ; Français, langue d'enseignement; Intégration linguistique scolaire et sociale, entre autres) (Ministère de l'éducation du Québec [MEQ], 2006 ; Ministère de l'Éducation, du Loisir et du Sport (MELS), 2014), l'utilisation de la littérature de jeunesse dans les classes québécoises, et plus particulièrement l'utilisation de l'album, est de plus en plus documentée dans les écrits scientifiques (Montésinos-Gelet et coll., 2018). Vu la myriade de sujets et de thématiques abordés à travers les albums de littérature jeunesse, les possibilités d'exploitation qui mènent à des contextes d'apprentissage signifiants et engageants pour les élèves sont multiples. De la classe ordinaire à la classe d'accueil, du préscolaire au secondaire, les enseignant $\cdot e \cdot s$ ont recours à ce médium pour soutenir, entre autres, le développement des compétences disciplinaires de différents domaines d'apprentissage du PFÉQ comme les langues, l'univers social ou les mathématiques (Turgeon, 2019 ; Boulet et Martel, 2017).

L'utilisation de l'album contribue également à poursuivre la mission de l'école québécoise selon laquelle l'élève doit faire l'apprentissage du vivre-ensemble, de la démocratie et de l'équité, attitudes et valeurs nécessaires pour évoluer dans une société pluraliste et diverse comme le Québec (MEQ, 2006). Lorsqu'encadrée et dirigée, la discussion autour d'albums abordant des questions éthiques et sociales peut contribuer chez les élèves, jeunes et moins jeunes, au développement d'un esprit critique et d'attitudes d'écoute et de respect de l'Autre (Armand et coll., 2015 ; Landry, 2019). Par exemple, en exploitant un album traitant de la différence, l'enseignant·e peut soutenir les élèves dans des discussions respectueuses sur l'exclusion, l'acceptation et le savoir vivre ensemble. C'est notamment de cette façon que, dès le préscolaire, les enfants peuvent développer des capacités de raisonnement moral, de décentration et d'empathie (Armand, 2014).

\section{Les critères pour le choix des Albums plurilingues ÉLODiL}

Ainsi, c'est avec l'objectif d'amorcer chez de jeunes enfants ces réflexions morales en lien avec la décentration, l'empathie et l'acceptation qu'ont été sélectionnés certains des albums de l'application. Par exemple, la différence physique est un thème présent dans L'ours brun qui voulait être blanc (Leroy et Delaporte, 2018), Poils aux pattes (Chabbert et Delaporte, 2018) et La petite feuille jaune (Cohen et Abesdris, 2015). Ces albums portent aussi sur le rejet engendré par cette différence et sur l'acceptation de soi et de l'Autre. Dans le même 
ordre d'idées, un classique québécois en matière de littérature jeunesse, Je suis terrible (Gravel, 2011), amène à dépasser les " premières impressions » face à un être différent (un monstre) qui pourrait susciter la peur et le rejet. Par ailleurs, deux albums, dont un dit «résistant », traitant du thème des émotions a été choisi en vue de permettre aux enfants une réflexion les guidant vers une meilleure connaissance de soi, comme le requiert le programme de l'éducation préscolaire. Les albums résistants sont ceux dont le texte ne donne pas nécessairement de réponse explicite aux questions du lecteur. Ces albums amènent donc celui-ci à réfléchir, le secouent, le laissent perplexe. Ainsi, alors que Je suis un raton laveur (Delporte, 2013) parle du chagrin et de la dépression chez une enfant, Un verger dans le ventre (Boulerice et Dubois, 2013) aborde les peurs et l'anxiété. Je suis un raton laveur fait d'ailleurs partie de la collection Coup de poing, constituée de livres de différentes maisons d'édition abordant des sujets délicats et qui ébranlent (Duguay, 2010). Enfin, sur une note plus légère, La Chicane (Anfousse, 2009), L'Abominable (Chaperon et Iris, 2013) et L'agneau qui voulait être un loup (Leroy et Delaporte, 2017) permettent aussi de traiter de la thématique des émotions et des sentiments, sous l'angle de l'amitié, à travers la présentation de situations de conflit et de rejet, mais aussi d'unité et de solidarité.

La littérature jeunesse étant un objet riche pour permettre aux lecteur-trice's de découvrir le monde et des réalités culturelles diverses (Dupin de Saint-André et Montésinos-Gelet, 2014), un autre des critères ayant guidé la sélection des 11 albums était la présence de repères culturels québécois en trame de fond des albums. Sans tomber dans le piège d'une représentation culturelle folklorique, le but était de permettre aux familles, en milieu pluriethnique et plurilingue, de développer des connaissances sur la société québécoise. Ce faisant, plusieurs des albums de l'application, soit Un verger dans le ventre (Boulerice et Dubois, 2013), Collation d'hiver (Dufresne et Béha, 2018), Une visite inattendue (Rioux et Dumont, 2018) et La Chicane, permettent de discuter des caractéristiques et des spécificités, au Québec, des saisons (la cueillette de pommes, les feuilles qui tombent en automne, les tempêtes de neige en hiver), des animaux (un écureuil, un pigeon, une moufette) ou encore de l'école (l'horaire, l'environnement physique).

Afin de soutenir l'émergence de l'écrit selon des approches plurilingues (littératies plurilingues), ces albums écrits originalement en français ont été traduits dans plusieurs langues. Les lecteur-trice $s$ en apprentissage du français langue seconde peuvent ainsi accéder à une compréhension plus fine de ces histoires, lorsqu'elles et ils ont la possibilité de lire et d'écouter l'histoire dans une autre langue connue au moyen de l'accès à distance à l'application. Par ailleurs, cette application ouvre la porte à l'exploration de la diversité linguistique à travers la traduction d'une même histoire dans plusieurs langues. Dans la prochaine section, après avoir exposé la pertinence de recourir à des albums bi/plurilingues, nous détaillons les modalités entourant la conception des Albums plurilingues ÉLODiL.

\section{Les albums bi/plurilingues}

Les albums bi/plurilingues sont ceux à l'intérieur desquels deux langues ou plus sont présentes. D'un album à l'autre, celles-ci peuvent être représentées en proportions variables et selon différentes configurations (Deschoux, 2017 ; Moore et Sabatier, 2014, Fleuret et Sabatier, 2018 ; Perregaux, 2009). Dans certains cas, les langues sont présentes dans des proportions équivalentes, sous forme de traduction systématique (un même texte traduit intégralement dans une autre langue ou plus). C'est le format qui a été privilégié pour les Albums plurilingues ÉLODiL.

L'album bi/plurilingue permet, à l'intérieur d'un même objet, la cohabitation de différentes langues, lesquelles sont susceptibles de jouir de statuts et de légitimité inégales compte tenu, notamment, des représentations sociales qui les accompagnent (Castellotti et Moore, 2002). L'album bi/plurilingue, selon sa configuration bien entendu, permet donc une représentation visuelle plus égalitaire entre les langues. Pour les enfants en apprentissage du français dans la société d'accueil, il s'agit d'un aspect qui revêt une grande importance. En effet, « chez certains enfants, la non-reconnaissance de l'existence de la langue de la famille, différente de celle de l'école, peut se traduire par une "insécurité linguistique", un sentiment de discrimination, une baisse de l'estime de soi, ainsi que par des difficultés à transférer des acquis cognitifs et langagiers d'une langue à l'autre » (Armand et coll., 2008, p. 47). À l'inverse, si le milieu de l'enfant reconnait l'ensemble des langues de son répertoire comme des outils de pensée et de communication complémentaires, créant ainsi des situations de bilinguisme additif, il lui sera plus facile de s'engager dans ses apprentissages, à la fois cognitivement et affectivement (Cummins, 2009). Ces propos démontrent bien, d'une part, l'importance de lutter contre l'imposition d'une norme monolingue (Armand, 2021; Lory et Armand, 2016) appliquée dans certains milieux scolaires. D'autre part, ils illustrent la nécessité de reconnaitre et de tirer profit de la diversité linguistique et culturelle par l'entremise, par exemple, d'approches plurilingues (Candelier, 2008; Moore, 2006) ou de pédagogies de translanguaging (García et Kleifgen, 2010) qui, encourageant les élèves à utiliser la totalité de 
leur répertoire, favorisent le bien-être et soutiennent les apprentissages. La mise en place de pratiques pédagogiques de translanguaging, dans lesquelles l'utilisation des langues du répertoire des élèves vise à soutenir les apprentissages, tant linguistiques que disciplinaires (ex : mathématique, sciences, etc.), permetle développement et la démonstration d'une conscience métalinguistique et sociolinguistique, en plus de les amener à s'engager activement dans les activités proposées (Cenoz et Gorter, 2015).

Par ailleurs, la mise en place d'activités pédagogiques et l'utilisation de supports qui permettent la mise en contact avec des langues variées contribuent à favoriser chez l'ensemble des élèves une décentration et une ouverture à l'Autre, nécessaires à un meilleur vivre-ensemble. C'est d'ailleurs ce que visent les approches d'éveil aux langues (Armand et coll., 2008), qui s'inscrivent parmi les approches plurielles des langues et des cultures (Candelier, 2008). À travers le contact avec des corpus oraux et écrits dans différentes langues et la manipulation de ces corpus, l'éveil aux langues a pour objectif d'encourager le développement langagier de l'ensemble des élèves et, notamment, le développement de leurs capacités métalinguistiques, tout en soutenant une construction identitaire harmonieuse et une ouverture à la diversité linguistique et culturelle. Ce faisant, l'album bi/plurilingue, qui met en perspective deux langues ou plus, représente un support privilégié pour concevoir et animer des activités d'éveil aux langues et, par exemple, développer les capacités métalinguistiques des élèves au moyen de comparaisons entre les langues. Nous décrivons ci-dessous les critères ayant guidé le choix des langues des traductions des albums ainsi que la démarche suivie, puis nous présentons brièvement les jeux d'éveil aux langues développés pour l'application.

\section{Les langues des traductions (écrites et orales) des Albums plurilingues ÉLODiL}

Dans le désir de favoriser l'ouverture à la diversité linguistique chez l'ensemble des élèves et de reconnaitre et valoriser les langues du répertoire linguistique des enfants bi/plurilingues en émergence, les 11 albums de l'application ont été traduits dans 22 langues $^{7}$. Ainsi, dans l'application, chaque double page d'un album est présentée en version numérique dans sa langue originale, le français, et un menu déroulant permet d'accéder à la traduction dans la langue sélectionnée. Pour faciliter l'accès à la correspondance entre le texte en français et dans la langue traduite, le texte des albums a été découpé en segments (un paragraphe ou quelques phrases associées à une même idée). Une flèche pointe les segments du texte en français pour lesquels apparait, sous la double-page, la traduction dans la langue sélectionnée, tel qu'il est possible de le constater dans la Figure 1 qui présente un extrait de l'album Poils aux pattes (Chabbert et Delaporte, 2018) et sa traduction en bengali.

\section{Figure 1}

Extrait de l'application Les Albums plurilingues ÉLODiL

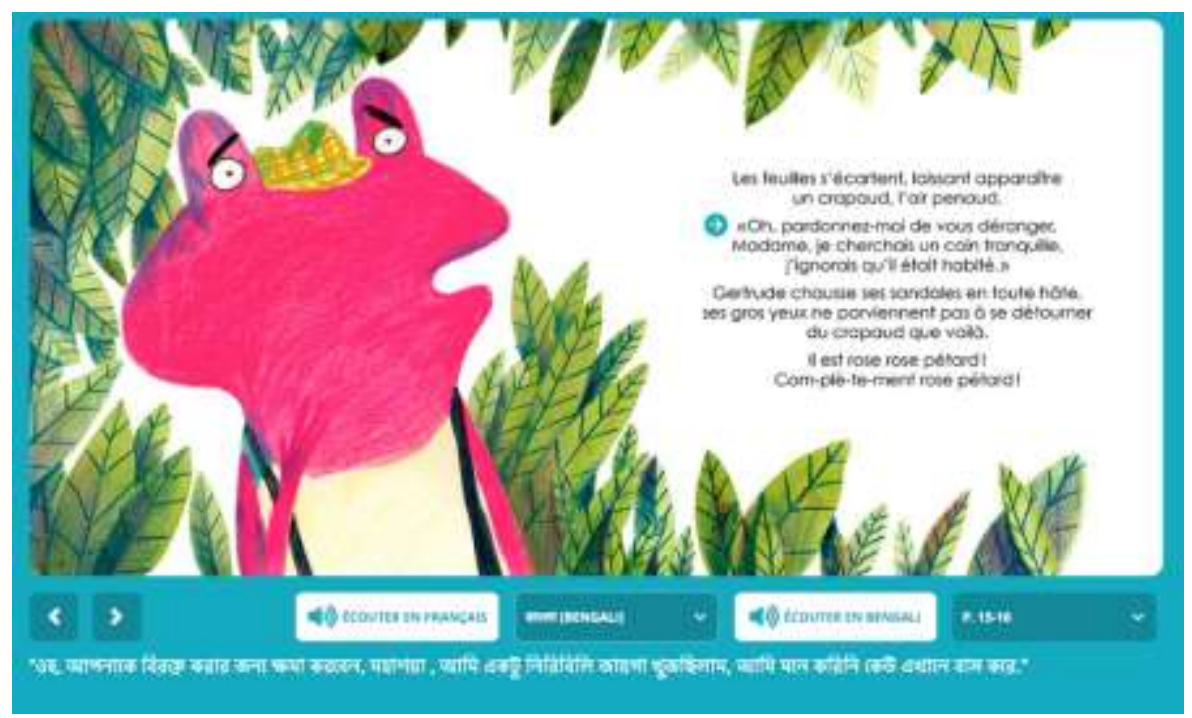

Note. L'album Poils aux pattes (Chabbert et Delaporte, 2018, p. 15-16)

Le choix des langues pour les traductions s'est fait en fonction des langues les plus parlées dans les écoles du Québec (par exemple, l'arabe, le créole, l'espagnol et le chinois). Une considération particulière a également 
été portée aux langues les plus parlées dans les écoles de Montréal et, plus spécifiquement, dans les quartiers où a été menée la recherche-action ayant donné naissance à l'application. Cela a mené au choix d'ajouter aux langues disponibles, par exemple, le bengali, le tamoul et le panjabi. Qui plus est, par souci de reconnaitre la diversité linguistique associée à la présence de nombreuses communautés autochtones au Québec, des traductions ont également été réalisées en atikamekw et en innu-aimun.

Les traductions ont été effectuées soit par des traducteur trice·s professionnel·le·s, soit par des intervenant $\cdot \mathrm{e} \cdot \mathrm{s}$ scolaires expert·e·s des langues en question. Enfin, dans l'application, les 11 albums sont présentés à l'oral, en français, grâce à des enregistrements faits en studio avec une conteuse professionnelle ${ }^{8}$. Par ailleurs, 7 des 11 albums, soit ceux qui ont été utilisés dans le cadre de la recherche-action, ont également été enregistrés à l'oral dans 10 langues $^{9}$ autres que le français, dont les langues les plus parlées par les élèves participants et leur famille. Les conteur·euse's ayant réalisé ces enregistrements ont ainsi eu l'occasion de réviser sommairement les traductions effectuées à l'écrit.

\section{Les jeux d'éveil aux langues disponibles sur les Albums plurilingues ÉLODiL}

Pour chacun des sept albums utilisés dans le cadre de la recherche-action et pour lesquels des enregistrements audios sont donc disponibles dans différentes langues, quatre sortes de jeux d'éveil aux langues ont été conçus ${ }^{10}$. Ces jeux sollicitent la discrimination auditive et visuelle de mots présents dans les albums, et ce, dans différentes langues et, parfois, dans différents systèmes d'écriture. Par exemple, le jeu Écoute et trouve l'image consiste à écouter un mot congénère (les congénères sont des mots de langues différentes ayant le même sens et sensiblement la même forme à l'oral et/ou à l'écrit) prononcé dans trois langues différentes et à choisir, parmi trois images, celle correspondant au mot en question.

\section{Méthodologie et résultats}

Afin de recueillir des données sur la fréquentation de l'application à travers le Canada ainsi que sur son utilisation et son appréciation par les utilisateur·trice·s, nous avons utilisé, d'une part, un « espion » numérique associé à l'application Google Analytics (Google Analytics) ${ }^{11}$ et, d'autre part, un sondage en ligne destiné aux enseignant·e.s et aux parents ayant utilisé l'application à distance pendant la pandémie ${ }^{12}$.

\section{Fréquentation de l'application (résultats obtenus grâce à l'espion numérique)}

L'analyse des données obtenues au moyen de l'espion numérique révèle que, entre le 21 avril et le 30 juin 2020 , 33269 « utilisateur·trice·s $\|^{13}$ ont fréquenté l'application, et ce, parfois à plus d'une reprise. En effet, un grand total de 58198 visites est recensé, lesquelles ont été effectuées au moyen d'ordinateurs (56,48\%) ou de tablettes et de téléphones intelligents (43,52\%). En moyenne, une visite de l'application a duré 8 minutes 32 secondes et a impliqué la consultation d'entre un et deux albums. Par ailleurs, l'application a été utilisée dans 398 villes canadiennes, dont la grande majorité se trouve au Québec. La ville comptant le plus grand nombre d'utilisateur·trice·s est Montréal (13 297), suivie de Laval (1 964), de Québec (1 578) et de Longueuil (1 144). Une plus haute fréquentation de l'application en semaine par rapport aux fins de semaine laisse penser que son utilisation a vraisemblablement été associée à des activités scolaires proposées par les enseignant·e·s.

Pour l'ensemble des albums, les cinq langues des traductions que les utilisateur-trice·s ont le plus fréquemment choisies pour lire les histoires sont, dans l'ordre, l'arabe, l'anglais, l'espagnol, l'atikamekw et le bengali (rappelons que les albums étaient tous présentés, par défaut, en français). Par ailleurs, en ce qui concerne l'écoute des histoires, l'analyse des langues sélectionnées révèle qu'elles ont le plus souvent eu lieu en français, mais que les autres langues proposées ont également été sélectionnées.

Dans l'ensemble, les 11 albums ont été consultés 108220 fois. Le nombre de consultations de chaque album varie grandement, et les quatre albums les plus lus et/ou écoutés sont L'agneau qui voulait être un loup (20 972 consultations), Je suis terrible (14 418 consultations), Une visite inattendue (13 872 consultations) et L'ours brun qui voulait être blanc (13 394 consultations). La plus fréquente consultation de L'agneau qui voulait être un loup pourrait être associée à sa mention dans un menu d'activités éducatives suggérées par le MEES et au fait qu'il s'agit du premier album qui apparait sur l'application. 


\section{Utilisation et appréciation de l'application (résultats obtenus grâce au sondage rempli par les enseignant $\cdot \mathbf{e} \cdot \mathbf{s})$}

Le sondage destiné aux enseignant·e·s, hébergé sur Lime Survey, a été partagé dans des courriels envoyés dans les milieux scolaires de même que sur la page Facebook ÉLODiL. Ce sondage compte 21 items, dont 11 à propos desquels les enseignant·e·s doivent indiquer leur degré d'accord (" tout à fait d'accord ", " d'accord ", « ni en accord, ni en désaccord ", « en désaccord " ou « tout à fait en désaccord ») et qui permettent de sonder leur appréciation de l'application en général. Ces items portent par exemple sur le choix des albums et de leurs thèmes, sur la présence des différentes langues, sur le potentiel de l'application pour soutenir les apprentissages langagiers des élèves et pour développer chez ces derniers des attitudes positives vis-à-vis de la lecture et de la diversité linguistique et, enfin, sur son potentiel pour favoriser l'établissement de liens avec les familles. Par la suite, les enseignant·e·s sont invité·e·s à préciser les manières dont l'application a été utilisée dans leur enseignement à distance et à partager des commentaires des élèves ou de leurs parents à propos de l'application. Enfin, six items sont d'ordre sociodémographique (ville et province de résidence, type de classe, niveau d'enseignement, caractéristiques du milieu d'enseignement [pluriethnique et/ou défavorisé]).

Il est à noter qu'un sondage similaire à celui conçu pour les enseignants·e·s a été diffusé à l'intention des familles. Toutefois, seuls sept parents ont répondu à l'appel, probablement en raison des canaux de diffusion du sondage, destinés surtout à des enseignant·e·s. Sans présenter en détail les résultats de ce deuxième sondage, nous reprendrons certains propos des parents lorsqu'ils viennent compléter les réponses des enseignant $\cdot \mathrm{e} \cdot \mathrm{s}$.

Mentionnons d'emblée que le sondage a été rempli par un échantillon restreint de 37 intervenant·e·s, soit 36 enseignant·e.s et une orthopédagogue de sept villes différentes du Québec et d'une ville en Ontario. Ces intervenant.e.s œuvrent dans différents contextes d'enseignement: classe d'accueil (18/37), classe ordinaire (14/37), classe d'adaptation scolaire (3/37), soutien linguistique (1/37), orthopédagogie (1/37); à différents niveaux: préscolaire $(7 / 37), 1^{\mathrm{er}}, 2^{\mathrm{e}}$, et $3^{\mathrm{e}}$ cycles du primaire $(27 / 37)$, secondaire $(3 / 37)$; et majoritairement dans des milieux pluriethniques et plurilingues (34/37) et variés sur le plan socioéconomique (27/37 en milieu défavorisé). Bien qu'il ne soit pas possible de généraliser les résultats obtenus en raison de la taille de l'échantillon, nous avons tout de même constaté une convergence dans les réponses des intervenant·e·s, que nous exposons dans les sections qui suivent.

Les réponses au sondage révèlent que les intervenant·e·s ont utilisé l'application de différentes façons, tantôt comme une ressource à diffuser auprès des familles en vue d'une utilisation sans intervention pédagogique, soit sans consigne particulière (20/37), tantôt comme une ressource à exploiter au moment d'un enseignement en mode synchrone, et ce, de différentes manières. Par exemple, les intervenant·e·s ont demandé à leurs élèves de lire un album en particulier et ont fait un retour sur celui-ci en séance synchrone (ex : Zoom, Teams, etc.) $(18 / 37)$ et/ou ont animé la lecture d'un ou de plusieurs albums en visioconférence (22/37).

Parmi les intervenant·e·s, 33 sur 37 étaient « tout à fait d'accord » ou « d'accord » pour affirmer que les Albums plurilingues ÉLODiL leur ont permis de se sentir plus outillé e.s pour soutenir les apprentissages de leurs élèves dans un contexte d'enseignement à distance. Les intervenant $\cdot e \cdot s$ déclarant travailler en milieu pluriethnique et plurilingue étaient aussi majoritairement "tout à fait d'accord » ou " d'accord » (30/32) ${ }^{14}$ pour affirmer que l'application a permis de soutenir, plus spécifiquement, l'apprentissage du français langue seconde auprès de leurs élèves. Les commentaires de l'ensemble des intervenant $\cdot e \cdot s$ permettent d'ailleurs de constater la diversité des activités pédagogiques menées à partir des albums de l'application pour soutenir différentes facettes du domaine langagier.

\section{Soutien à distance des apprentissages du domaine langagier en français}

À partir des albums et de leurs enregistrements audios, les intervenant·e·s ont animé une panoplie d'activités visant le développement des compétences Lire et écrire des textes variés et Apprécier des œuvres littéraires ainsi que l'acquisition de différentes connaissances de la progression des apprentissages en français, langue d'enseignement (MELS, 2009). Par exemple, des activités d'écriture à la suite d'une lecture ont été menées. Les élèves ont aussi été appelés à porter un jugement critique sur les albums à travers la production de résumés et l'appréciation de lectures à l'oral et à l'écrit. Quant à elle, la compréhension de textes a été travaillée à différents niveaux au moyen de l'étude du schéma narratif, de questions de compréhension et de la mobilisation de stratégies avant et pendant la lecture. Également, certaines activités ont visé le développement de la conscience phonologique des élèves (opérations métaphonologiques, étude des sons du français et travail sur la 
prononciation et la prosodie), du vocabulaire et de la grammaire (étude des types de phrases, accords au sein du groupe nominal, etc.).

\section{Soutien à distance de l'apprentissage du français en favorisant les transferts entre les langues}

Tou.te·s les intervenant·e·s étaient « tout à fait d'accord » ou "d'accord » pour affirmer qu'elles et ils ont apprécié que les Albums plurilingues ÉLODiL soient disponibles en français et dans d'autres langues, ce qui leur a notamment permis de soutenir, à distance, l'apprentissage du français au moyen d'approches d'éveil aux langues et de stratégies pédagogiques favorisant les transferts entre les langues. Par exemple, certain·e·s ont repéré avec leurs élèves des mots congénères entre les différentes langues ou ont demandé à leurs élèves de résumer en français une histoire lue dans une autre langue connue. Elles ou ils ont rapporté que les allersretours possibles entre les versions de l'histoire en français et dans différentes langues connues des élèves permettaient à ceux-ci de valider leur compréhension de l'histoire en français. Un·e des intervenant·e·s a par ailleurs mentionné au passage que « l'élève se sent valorisé lorsqu'il voit sa langue maternelle utilisée dans l'enseignement ", ce qui nous permet de constater que les Albums plurilingues ÉLODiL ont bien servi à légitimer les répertoires linguistiques des élèves et à créer des situations de bilinguisme additif. Mentionnons toutefois l'inquiétude d'un·e intervenante par rapport au fait que toutes les langues parlées par ses élèves ne se retrouvaient pas sur l'application, craignant ainsi que certains se sentent exclus.

Les jeux d'éveil aux langues disponibles sur l'application représentaient un autre espace privilégié pour favoriser les transferts entre les langues au moyen d'activités ludiques. Toutefois, nous constatons que ceux-ci ont peu été utilisés dans l'enseignement à distance : seulement quatre intervenant·e·s ont déclaré avoir échangé avec leurs élèves à propos des jeux. II est possible de penser que les intervenant·e·s ont préféré recourir à des activités pédagogiques planifiées en fonction des besoins de leurs élèves plutôt qu'à des jeux plus ludiques qui ont possiblement été exploités davantage du côté des familles.

\section{Ouvrir à la diversité linguistique et à l'Autre}

Le fait que les histoires des Albums plurilingues ÉLODiL soient disponibles en différentes langues laisse aussi croire que leur utilisation a entrainé chez les enfants un éveil et un intérêt par rapport aux différentes langues de l'application. Encore une fois en très grande majorité (30/37), les intervenant·e·s étaient « tout à fait d'accord » ou « d'accord » pour affirmer que les Albums plurilingues ÉLODiL ont permis de susciter chez leurs élèves une curiosité et une ouverture vis-à-vis de la diversité linguistique. L'extrait suivant, qui sous-entend un investissement de la part de l'enfant se traduisant par une curiosité et une posture réflexive par rapport aux langues, témoigne bien du fait que certains élèves ont démontré un intérêt pour des langues qu'ils ne connaissaient pas à priori : U Un élève m'a dit "Je faisais la lecture dans trois langues que je ne connaissais pas, puis je revenais à ma langue maternelle, le créole. Je terminais par le français. Parfois, on dirait que l'histoire était meilleure quand je ne comprenais pas les mots. D'autres fois, c'était le contraire." „Que certains élèves aient, de leur propre initiative, écouté les histoires dans des langues " qu'ils ne connaissaient pas » marque la potentielle contribution de l'application pour initier chez ceux-ci une décentration par rapport aux langues de leur répertoire, tant les langues familiales que celle de la scolarisation.

L'utilisation de l'application a aussi été l'occasion de discuter des langues en soi, des accents différents et du linguicisme. Nous pouvons penser que cela a contribué au développement d'attitudes d'ouverture et de tolérance vis-à-vis de la (ou des) langue(s) de l'Autre : "Certains riaient des accents, des langues. Nous avons pu discuter du fait qu'il est vrai que certains sons ou prononciations peuvent nous faire rire parce qu'ils sont nouveaux ou étranges pour nous, mais que nous devons demeurer ouverts et respectueux. " La possibilité de voir et surtout d'entendre différentes langues sur l'application aura donc permis à certain·e $\cdot s$ enseignant·e·s de travailler avec les élèves sur les attitudes et les représentations qui fondent l'ouverture à l'Autre et à la solidarité (Candelier, 2003).

Comme nous l'avons mentionné précédemment, les thèmes de certains albums, comme la différence, étaient également propices à des discussions d'ordre éthique, une occasion que certain $\cdot e \cdot s$ intervenant $\cdot e \cdot s$ ont saisie, comme nous le montrons dans les lignes qui suivent. 


\section{Appréciation des albums et plaisir de la lecture}

Toujours de façon unanime (35/37 « tout à fait en accord » ou « en accord »), les intervenant·es ont apprécié le choix des albums et les thèmes qu'ils abordaient. Quelques-un·e·s ont toutefois mentionné qu'elles ou ils auraient aimé avoir accès à une variété de types de livres (comme des albums documentaires) ou encore à des albums pour des élèves plus âgés. Rappelons que, conçue particulièrement pour le préscolaire et le $1^{\mathrm{er}}$ cycle, l'application a été utilisée jusqu'au secondaire.

Selon les propos des intervenant·e·s, les thèmes abordés dans les albums se prêtaient à des discussions et à des retours enrichissants ainsi qu'à l'établissement de liens avec le vécu des élèves, ces derniers étant ainsi mieux à même de réagir aux textes lus. Certain·e·s intervenant·e·s se sont par ailleurs engagé·e·s avec leurs élèves dans des discussions sur des sujets éthiques et moraux, contribuant de cette manière à favoriser des attitudes de vivre-ensemble chez les élèves : " J'ai utilisé l'album L'ours brun qui voulait être blanc pour discuter des évènements en lien avec le "Black Lives Matter". Nous avons eu une discussion sur le racisme, la différence et l'acceptation de soi. »

Finalement, une enseignante a mentionné que le fait que certains des albums ou des auteur·trice·s étaient connus des élèves a permis de renforcer leur intérêt pour la lecture. Dans cet ordre d'idées, la quasi-totalité des intervenant·e·s (36/37) a déclaré être " tout à fait en accord " ou " en accord " pour affirmer que leurs élèves ont apprécié lire et/ou écouter les histoires des Albums plurilingues ÉLODiL, voire que les albums de l'application leur ont donné davantage envie de lire et/ou d'écouter des histoires (32/37). Ces constats proviennent des commentaires positifs émis par les élèves à propos des albums et des témoignages d'enseignant·e·s concernant certains élèves qui attendaient impatiemment la « lecture du jour ».

\section{Soutien aux relations école-famille}

Les intervenant·e·s étaient en majorité (30/37) " tout à fait d'accord » ou " d'accord " pour dire que les Albums plurilingues ÉLODiL ont permis de renforcer ou d'établir des liens avec les familles, notamment celles issues de l'immigration (25/29). En effet, les commentaires qu'elles ou ils ont émis montrent que la ressource a été partagée aux parents qui ont beaucoup apprécié avoir accès à des livres numériques " agréables ", utilisés dans les activités scolaires de leurs enfants et accessibles gratuitement et dans différentes langues. Cela aurait notamment contribué à ce que certains parents se sentent impliqués dans la vie scolaire et les apprentissages de leurs enfants, même en dépit d'une possible connaissance limitée du français. L'application leur aurait donc permis de déployer activement leurs ressources linguistiques.

Également, les commentaires des enseignant.e.s révèlent que les Albums plurilingues ÉLODiL ont voyagé à l'intérieur des familles, tel que souhaité, menant à des interactions familiales autour de la littératie et légitimant, par le fait même, leur capital linguistique : « Ils aimaient lire les livres avec leurs petits frères et petites sœurs. Un de mes élèves m'a dit qu'il lisait des livres en créole pour la première fois et que toute sa famille participait à la lecture. » Encore une fois, nous constatons que l'application a permis de créer des situations où, à la fois la famille et l'école reconnaissent et légitiment les différentes langues de l'enfant, ouvrant ainsi la porte à des pratiques de translanguaging. Par ailleurs, l'application a aussi entrainé des discussions familiales autour des langues, et plus particulièrement autour des variétés d'une même langue : « Un de mes élèves était plutôt surpris d'entendre l'histoire en arabe, mais son papa a dit que ce n'était pas l'arabe qu'ils parlaient, que c'était un arabe "académique" ».

Il est intéressant de constater, grâce au sondage diffusé auprès des parents, que ces discussions autour des langues ont également eu lieu au sein de quelques familles décrivant elles-mêmes leur environnement langagier comme majoritairement francophone. Par exemple, les propos d'un parent révèlent que l'application a créé une occasion de discuter du concept de langues et qu'elle a réellement suscité un intérêt et une curiosité pour les langues chez une enfant âgée de trois ans et demi :

Elle saisit le concept des langues, fait semblant d'en parler différentes. Je pense qu'elle a surtout apprécié de voir une histoire qu'elle connaissait et aimait déjà racontée dans une langue qu'elle ne comprenait pas. Du moins, à cause de notre réalité, c'est surtout l'utilisation que nous avons faite de l'outil : exposer notre enfant à des sonorités différentes, lui montrer que des langues différentes existent et qu'elles peuvent aussi raconter la même histoire. 


\section{Conclusion}

Dans le cadre de cette période d'enseignement à distance imposée par des circonstances exceptionnelles, nous pouvons penser, en dépit du nombre limité de répondant·e·s au sondage, que l'accès à l'application d'albums plurilingues a permis d'engager élèves, familles et enseignant $\cdot e \cdot s$ dans un processus signifiant d'enseignementapprentissage du français. Elle s'avère une ressource prometteuse pour soutenir un enseignement de qualité et respectueux des ressources des élèves et de leurs familles.

L'application représente également un outil intéressant pour les familles qui n'ont pas nécessairement toujours accès à une variété de livres dans leurs langues maternelles (Armand et coll., 2005), surtout lorsqu'il est question de langues « minoritaires ». Ainsi que le souligne Perregaux (2009, p.131): " l'édition reproduit la hiérarchie linguistique économique sauf quand elle fait preuve d'un volontarisme affiché de visibiliser des langues souvent absentes du champ littéraire habituel, ou d'une position politique qui veut sortir de l'anonymat et de la tendance à la sous-catégorisation des langues utilisées par de nombreux locuteurs, comme les langues de la population migrante. ". Dans le cadre de sa mise en ligne ouverte à tou·te $\cdot \mathbf{s}$, l'application a permis à tous les individus qui ont navigué sur celle-ci d'être exposés à la diversité linguistique et, notamment, à des langues souvent « invisibilisées » dans les écrits. Cela a également permis à des parents d'ouvrir un dialogue familial à propos des langues, un des aspects d'une éducation citoyenne et interculturelle nécessaire pour évoluer dans le monde d'aujourd'hui.

Le potentiel de l'application pour soutenir des liens plus étroits entre les familles, l'école et la communauté reste à explorer. À cet effet, il serait intéressant que l'école et les familles réfléchissent ensemble aux modalités d'articulation d'une collaboration qui tiendraient compte des pratiques et des intérêts des familles autour de la lecture partagée d'albums de littérature jeunesse plurilingues. Dans le même esprit, en milieu communautaire, des ateliers parents-enfants autour de la lecture partagée des albums de l'application, dont les modalités sont aussi à explorer, pourraient permettre de soutenir l'apprentissage du français, tout en prenant appui sur les différentes ressources du répertoire linguistique des familles et en soutenant l'entrée dans l'écrit des enfants.

Pour terminer, tou·te-s les intervenant·e·s, sans exception, ont affirmé souhaiter continuer de se servir de l'application dans leur enseignement. À la lumière de leurs commentaires et vu l'appréciation et l'utilité de l'application auprès d'élèves de différents niveaux, il serait intéressant d'y intégrer éventuellement plus de langues ainsi que d'œuvres de littérature jeunesse, notamment de différents types, lesquelles pourraient aussi être destinées à un public plus large en termes d'âge.

Depuis septembre 2021, les Albums plurilingues ÉLODiL sont accessibles gratuitement dans toutes les bibliothèques scolaires du Québec via le projet Biblius ${ }^{15}$, une plateforme de prêt de livres numériques consacrée au milieu scolaire québécois (déployée dans le cadre du Plan d'action numérique en éducation du ministère de l'Éducation du Québec). Par ailleurs, les fiches pédagogiques produites pour les 7 albums qui ont servi à la recherche-action seront également accessibles sur cette plateforme.

\section{Notes}

${ }^{1}$ Le terme répertoire linguistique est utilisé par les sociolinguistes pour caractériser « l'ensemble de variétés linguistiques - plus ou moins bien maitrisées ou développées - que possède un acteur social donné et dont il peut jouer, selon les situations, en faisant appel à telle ou telle de ces variétés » (Coste, 2002, p.117). ॥ s'agit en somme de l'ensemble des compétences langagières et des connaissances linguistiques d'un individu.

2 Nous remercions la Direction de l'intégration linguistique et de l'éducation interculturelle (DILEI) du ministère de l'Éducation du Québec (MEQ) pour le financement de l'application ainsi que Benoit Dubuc et son équipe (Espace Courbe, puis Toumoro) pour le soutien technique à sa conception.

${ }^{3}$ Nous remercions la DILEI (MEQ) et les maisons d'édition québécoises La Courte échelle et Les 400 Coups qui ont permis la diffusion gratuite de l'application à travers le Canada après la fin du projet de recherche-action.

${ }^{4}$ Les 7 albums utilisés dans le cadre de la recherche-action sont les suivants (voir ci-dessous pour la référence complète) : Je suis terrible, L'ours brun qui voulait être blanc, Une visite inattendue, Poils aux pattes, Un verger dans le ventre, La petite feuille jaune, L'agneau qui voulait être un loup.

${ }^{5}$ Nous remercions l'organisme JAME (http://www.jame-mtl.org/) pour cette collaboration. 
${ }^{6}$ Nous remercions Benoit Dubuc pour sa participation au traitement des données de Google Analytics.

${ }^{7}$ Ces 22 langues sont les suivantes : anglais, arabe, atikamekw, bengali, chinois, créole haïtien, espagnol, grec moderne, innu-aimun, italien, kabyle, ourdou, panjabi, persan, portugais, roumain, russe, swahili, tagalog, tamoul, turc et vietnamien.

${ }^{8}$ Nous remercions Suzanne Côté, qui a prêté sa voix à ces albums.

${ }^{9}$ Les 10 langues sont les suivantes : anglais, arabe, bengali, créole, espagnol, italien, ourdou, panjabi, russe et tamoul.

${ }^{10}$ Nous remercions Toumoro pour la conception des jeux et Ismaël Seck pour sa contribution technique.

${ }^{11}$ Soulignons les limites de l'outil dans la mesure où certains navigateurs bloquent le transfert de données vers Google Analytics.

12 L'obtention de ces données respecte pleinement l'anonymat de tou'te $\cdot s$ les participant·e.s.

${ }^{13}$ Chaque « utilisateur·trice » correspond à un appareil différent ayant permis de fréquenter l'application.

${ }^{14}$ Sur les 34 enseignant·e.s ayant affirmé œuvrer en milieu pluriethnique et plurilingue, 32 ont répondu à la question.

${ }^{15}$ Pour plus d'information sur le projet : https://projetbiblius.cal. Nous remercions la DILEl qui a octroyé le contrat pour le transfert de l'application sur la plateforme Biblius. 


\section{Bibliographie}

Armand, F. (2021). Enseignement du français en contexte de diversité linguistique au Québec : idéologies linguistiques et exemples de pratique en salle de classe. Dans M. Potvin, M.-O. Magnan et J. LarochelleAudet et J.-L. Ratel (dir.), La diversité ethnoculturelle, religieuse et linguistique en éducation au Québec. Théorie et pratique ( $2^{\mathrm{e}}$ éd., p. 232-244). Fides Éducation.

Armand, F. (2014). Pourquoi l'éducation inclusive en maternelle ? Les ieunes enfants sont-ils « racistes »? Revue préscolaire, 52(3), 21-23.

Armand, F., Dagenais, D. et Nicollin, L. (2008). La dimension linguistique des enjeux interculturels : de l'Éveil aux langues à l'éducation plurilingue. Rapports ethniques et éducation : perspectives nationales et internationales, 36(1), 44-64.

Armand, F., Gagné, J., De Koninck, Z. et Dutil, C. (2005). Exploration des pratiques de littératie en milieu familial et portrait démolinguistique d'élèves immigrants allophones nouvellement arrivés en situation de grand retard scolaire au Québec. Canadian Journal of Applied Linguistics, 8(1), 7-26.

Armand, F., Gosselin-Lavoie, C. et Combes, E. (2016). Littérature jeunesse, éducation inclusive et approches plurielles des langues. Nouvelle Revue Synergies Canada, 9, 1-5.

Armand, F., Gosselin-Lavoie, C. et Maynard, C. (2018). Les Albums plurilingues ÉLODiL. Application réalisée dans le cadre d'un contrat de la DILEI (MEQ). Disponible à partir de septembre 2021 sur la plateforme Biblius (Bibliopresto).

Armand F., Gosselin-Lavoie, C., Turgeon E., Audet G., Borri-Anadon C. et Charette J. (2017-2021). Favoriser le développement de l'oral et l'entrée dans l'écrit chez les enfants à l'éducation préscolaire en milieu pluriethnique et plurilingue au moyen de la lecture partagée et d'approches plurilingues, Rapport déposé en juin 2021, FRQSC - Actions concertées lecture et écriture.

Armand, F., Pelletier, M., St-Hilaire, L. et Gosselin-Lavoie, C. (2015). Littérature jeunesse et interactions orales. Discuter de la diversité linguistique et culturelle, de la différence, du rejet, du racisme.... Québec français, 174, 21-23.

Boulet, M. et Martel, V. (2017). Enseigner de façon interdisciplinaire l'univers social au premier cycle du primaire grâce à la littérature jeunesse. Vivre le primaire, Hiver, 28-31.

Candelier, M. (2003). Evlang : les enjeux. Dans M. Candelier (dir.), L'éveil aux langues à l'école primaire (p. 19-38). De Boeck Supérieur.

Candelier, M. (2008). Approches plurielles, didactiques du plurilinguisme : le même et l'autre. Recherches en didactique des langues et des cultures, 1(5). http://journals.openedition.org/rdlc/6289, doi.org/10.4000/rdlc.6289.

Castellotti, V. et Moore, D. (2002). Représentations sociales des langues et enseignements. Guide pour l'élaboration des politiques linguistiques éducatives en Europe - De la diversité linguistique à l'éducation plurilingue. Étude de référence pour le Conseil de l'Europe. https://www.researchgate.net/publication/255602221 REPRESENTATIONS SOCIALES DES LANGU ES ET ENSEIGNEMENTS Guide pour l'elaboration des politiques linguistiques educatives en Eur ope - De la diversite linguistique a l'education plurilingue.

Cenoz, J. et Gorter, D. (dir.). (2015). Multilingual Education: Between language learning and translanguaging. Cambridge University Press.

Comité de gestion de la taxe scolaire de l'ile de Montréal (CGTSIM). (2020). Portrait socioculturel des élèves inscrits dans les écoles publiques de l'île de Montréal - inscriptions au 8 novembre 2019. https://www.cgtsim.qc.ca/fr/documents-site-web/564-portrait-socioculturel-inscrits-2019-11-08/file. 
Cummins, J. (2009). Transformative multiliteracies pedagogy: School-based strategies for closing the achievement gap. Multiple Voices for Ethnically Diverse Exceptional Learners, 11, 38-56.

Deschoux, C. -A. (2017). Passer les frontières : apport des albums bilingues pour la jeunesse. Educateur (L'), 9, 14-16.

Duguay, M. (2010, septembre). Un projet qui ébranle les élèves. Vie pédagogique, 155, 44-46.

Dupin de Saint-André, M. et Montésinos-Gelet I. (2014). La diversité culturelle au sein d'œuvres de jeunesse. Québec français, (172), 69-71.

Fleuret, C. et Sabatier, C. (2018). La littérature de jeunesse en contextes pluriels : perspectives interculturelles, enjeux didactiques et pratiques pédagogiques. Français dans le monde. Recherches et applications, 65, 95-111.

García, O. et Kleifgen J.-A. (2010). Educating emergent bilinguals: policies, programs, and practices for English language learners. Teachers College Press.

Gosselin-Lavoie, C. et Armand, F. (2015). Favoriser l'entrée dans l'écrit chez les jeunes enfants allophones. Revue canadienne des jeunes chercheures et chercheurs en éducation, 6(2), 94-101.

Hornberger, N. H. (2001). Multilingual Literacies, Literacy Practices, and the Continua of Biliteracy. Dans M. Martin-Jones et K. E. Jones (dir.), Studies in Written Language and Literacy, 10, 353. John Benjamins Publishing Company. DOI:10.1075/swll.10.29hor.

Lambert, W. E. (1975). Culture and Language as Factors in Learning and Education. Dans A. Wolfgang (dir.), Education of Immigrant Students (p. 23-61). The Ontario Institute for Studies in Education.

Landry, H. (2019). Les effets d'une séquence d'enseignement-apprentissage de l'oral inspirée des approches de la philosophie pour enfants et des approches plurilingues sur la compétence discursive d'élèves du 3e cycle du primaire en classe d'accueil. [Mémoire de maîtrise inédit]. Université de Montréal. http://hdl.handle.net/1866/23570

Lory, M.-P. et Armand, F. (2016). Éveil aux langues et évolution des représentations d'élèves plurilingues sur leur répertoire linguistique. Alterstice, 6(1), 27-38. DOI:10.7202/1038276ar

Ministère de l'Éducation, du Loisir et du Sport (MELS). (2014). Enseignement primaire : intégration linguistique, scolaire et sociale.

http://www.education.gouv.qc.ca/fileadmin/site web/documents/education/jeunes/pfeg/PFEQ integration -linguistique-scolaire-sociale-primaire 2014.pdf

Ministère de l'Éducation, du Loisir et du Sport (MELS). (2009). Progression des apprentissages au primaire : Français langue d'enseignement. Programme de formation de l'école québécoise. http://www.education.gouv.qc.ca/fileadmin/site web/documents/education/jeunes/pfeq/PDA PFEQ fran cais-langue-enseignement-primaire 2011.pdf

Ministère de l'éducation du Québec (MEQ). (2006). Programme de formation de l'école québécoise : Éducation préscolaire, enseignement primaire. Québec : Ministère de l'éducation, du loisir et du sport. http://www4.banq.qc.ca/pgq/2006/3127968.pdf

Montésinos-Gelet, I., Leclaire-Halté, A., Maisonneuve, L., Scheepers, C., Delneste, S., Turgeon, E., Lépine, M., Hébert, M., Charron, A., Dupin de Saint-André, M., Colognesi, S., Deschepper, C. et DuvinParmentier, B. (2018). Pleins feux sur l'album. Centre de diffusion et de formation en didactique du français de l'Université de Montréal.

Moore, D. (2007). Plurilinguismes et école. Didier. 
Moore, D. et Sabatier, C. (2014). Les approches plurielles et les livres plurilingues. De nouvelles ouvertures pour l'entrée dans l'écrit en milieu multilingue et multiculturel. Nouveaux cahiers de la recherche en éducation, 17(2), 32-65. DOI :https://doi.org/10.7202/1030887ar

Perregaux, C. (2009) Livres bilingues et altérité. Nouvelles ouvertures pour l'entrée dans l'écrit. Figurationen, 10(12), 127-140.

Rodriguez-Valls, F. (2009). Cooperative Bi-Literacy: Parents, Students, and Teachers Read to Transform. English Teaching: Practice and Critique, 8(2), 114-136.

Swain, M. (1985). Communicative competence: Some roles of comprehensible input and comprehensible output in its development. Dans S. M. Gass et C.G. Madden (dir.), Input in second language acquisition (p. 165-179). Newbury House Publishers.

Turgeon, É. (2019). Adopter des conduites interprétatives de 4 à 6 ans : quelle progression ?. Repères, 59, 5170. DOI: $10.4000 /$ reperes.2021

\section{Annexe - Liste des albums de l'application}

Anfousse, G. (2009). La chicane. La Courte échelle.

Boulerice, S. et Dubois, G. (2013). Un verger dans le ventre.

Chabbert, I. et Delaporte, B. (2018). Poils aux pattes.

Chaperon, D. et Iris. (2013). L'abominable. La Courte échelle.

Cohen, L. et Abesdris, M.-A. (2019). La petite feuille jaune.

Delporte, J. (2013). Je suis un raton laveur. La Courte échelle.

Dufresne, R. et Béha, P. (2018). Collation d'hiver.

Gravel, É. (2011). Je suis terrible. La Courte échelle.

Leroy, J. et Delaporte B. (2017). L'agneau qui voulait être un loup.

Leroy, J. et Delaporte B. (2018). L'ours brun qui voulait être blanc.

Rioux, A.-M. et Dumont, Y. (2018) Une visite inattendue. 